\title{
Extended musicking, extended mind, extended agency. Notes on the third wave
}

\author{
Kevin Ryan a, b, *, Andrea Schiavio ${ }^{c}$ \\ a Department of Philosophy, University of Memphis, USA \\ ${ }^{\mathrm{b}}$ Institute for Intelligent Systems, University of Memphis, USA \\ c Centre for Systematic Musicology, University of Graz, Austria
}

\author{
This is a pre-print version - please cite original
}

\section{A R T I C L E I N F O}

\section{Keywords:}

Extended mind

Music cognition

Musicking

Music emotion

Music performance

\begin{abstract}
A B S T R A C T
The conceptual resources of ' $4 \mathrm{E}$ ' music cognition - i.e. the embodied, embedded, extended, and enactive paradigms - have offered a rich set of tools to explore the nature of musical experience. Among these four approaches, the extended mind perspective has heretofore received less overall attention. In this paper we focus on further developing the musically extended mind - especially in regards to musical performance - drawing on recent third wave developments. After exploring the main tenets of first wave (parity between internal and external components), second wave (complementarity between internal and external components), and third wave (dynamically changing internal and external components, as well as extended and decentralized agency) accounts of the extended mind, we turn to introducing existent first and second wave positions on music cognition. While they offer important insights, we suggest that elements of the third wave, especially focused on decentralized agency, are needed to capture the complexities of musical performance. We apply the third wave tools to the case of music performance in order to show, first, the specific limitations of the first two waves focus on parity and complementarity and, second, how a third wave account may be developed by applying it within this particular context.
\end{abstract}

\section{Introduction}

A growing number of contributions at the intersection of music and cognitive science have recently promoted the view that brain, body, and world are coupled in ways that require new tools to fully capture how we engage with, and make sense of, music (e.g., Clarke, 2005; Krueger, 2015, 2018; Leman, 2007; Loaiza, 2016; Reybrouck, 2005). These approaches are often grouped under the $4 E$ cognition umbrella - a label that indicates the fundamentally (i) Embodied, (ii) Embedded, (iii) Enactive, and (iv) Extended nature of mental life. Through the 4E lenses, cognition (i) cannot be taken as separate from particular facts of the bodies we have, (ii) is scaffolded by the environment, (iii) is geared towards action in the world with a continuum between life and mind, and (iv) extends outside the skull-skin boundary to loop into the world.

On a general level, it has been argued that the traditional focus on head-bound processes (representations, computations, etc.), besides being mostly concerned with cognitive mechanisms internal to an agent, can offer only limited accounts of what musical experience really en- tails (e.g., Reybrouck, 2012). While such frameworks have contributed important understandings concerning some aspects of the musical mind, some proponents of $4 \mathrm{E}$ cognition have argued they need to be traded for more flexible understandings of how agents across different cultural contexts actively participate in musical activities (Blacking 1973; Moran, 2014). Making this change may lead to new possibilities for the development of critical scholarship, practice, and empirical work (van der Schyff, Schiavio, Walton, Velardo, \& Chemero, 2018, 2016; Walton, Richardson, Langland-Hassan, \& Chemero, 2015). A significant feature of this shift is the renewed centrality of performance for the impressively wide range of states, feelings, and (inter)activities associated with the word 'music'.

At the same time, it may be hard - if not impossible - to propose a definition of 'music' that truly captures the importance and complexity of performance across different times, places, and cultures. In particular, understanding the term 'music' as a noun may downplay its inherently active and inter-relational roots. Therefore, we refer to 'music' as a synonym of 'musicking', or 'to music'. This verbal form was coined

\footnotetext{
* Corresponding author. Department of Philosophy, University of Memphis, USA.

Email address: kjryan1@memphis.edu (K. Ryan)
} 
by Christopher Small (1998) in the hope of providing a less static way to understand musical thought and action: in his words: "to music is to take part, in any capacity, in a musical performance, whether by performing, by listening, by rehearsing or practicing, by providing material for performance (what is called composing), or by dancing" (Small, 1998, p. 9, emphasis in original). The move is particularly useful when considering how apparently 'passive' practices such as listening to music, imagining music, or composing music, in fact, involve states where the whole body may resonate with the music (e.g., Schiavio, Menin, \& Matyja, 2015), and where we actively participate in its perception and fruition. Aligning with such insights, new approaches and theoretical work in music education (Borgo, 2005; Elliott \& Silverman, 2015), music performance (Clarke \& Doffman, 2017; Hayes, 2017; Parncutt \& McPherson, 2002; Whalley, Mavros, \& Furniss, 2014; Williamon, 2004), musical interaction (Clayton, 2017; Himberg, 2017), and music theory (De Souza, 2017; Kozak, 2015) have emerged, which increasingly draw from the 4E research paradigms (see Menary, 2010).

In this paper we aim to explore what the extended mind thesis can offer for research in music cognition, with a primary focus on the context of musical performance, along with additional insights concerning musical emotions.

In order to identify (dis)continuities between musical and cognitive levels, we will draw on both existing literature in music cognition and relevant considerations in the cognitive sciences at large. We maintain that investigating musical experience through a framework that has roots in philosophy of mind and cognitive science is not simply a theoretical exercise. Instead, it could provide important benefits in terms of promoting interdisciplinary dialogues between humanities and sciences, developing conceptual tools that may inspire new empirical research, and stimulating fruitful discussion in diverse practice-oriented musical contexts. Similarly, we suggest that extended approaches can help us capture features and dynamics that escape from more traditional views in music psychology and have not yet been fully explained by enactive, embodied, and embedded approaches.

The paper is structured as follows. In the next section we will describe specific research and theory on the extended mind thesis from the fields of cognitive science and music psychology. While all defenders of the extended mind thesis agree with this general idea, the sorts of arguments they employ differ in important ways. To navigate the complexities of the arguments being offered, a view of three different 'waves' has been adopted (see Sutton, 2010; Kirchoff, 2012). Here the arguments behind the proposal that cognitive processes may span across brain, body, and world to fluidly and fluently include elements outside of the skull-skin boundary will be analyzed in detail (Clark, 2008; Clark \& Chalmers, 1998; Sutton, 2010). Afterwards, we will discuss the three waves of the extended mind within the musical domain, drawing in particular from work by Tom Cochrane and Luke Kersten (1st wave), and Joel Krueger (2nd wave) who have already developed important understandings of how the extended mind approach can be carried out in musical contexts. In doing so, however, we wish to note here that these authors did not explicitly adhere to a particular wave of the extended mind. That is to say, for instance, Cochrane and Kersten do not claim to be defending a first wave account instead of a second (or third) wave account. Any overly clean distinction between different waves is more of an abstraction than a strict division among theories, since all proponents are ultimately arguing in defense of the same core claim that the mind is not localized inside the head. At the same time, as noted by John Sutton (2010), the wave metaphor is useful since it highlights important differences in how proponents of the extended mind thesis have grounded their arguments (see Fig. 1). We therefore maintain that these accounts can be usefully categorized by this metaphor, and that doing so will make the stakes of adopting a third wave account clearer.
After this overview, we will develop original insights on a third wave of the extended musical mind in terms of concrete musical activities and contexts. The aim of this section is to show how the third wave account of extended mind is better suited to capture the complexity of the musical events being described when compared to first and second wave alternatives. To conclude, we briefly consider additional possible applications for musical contexts and future research agendas.

\section{Extended (musical) mind: the three waves}

\subsection{A general taxonomy}

While there are multiple historical antecedents to the idea of the $e x$ tended mind, the locus of recent scholarship can be traced primarily back to work by Clark and Chalmers (1998). Here the authors, in part following Hillary Putnam's and Tyler Burge's semantic externalism - a position that holds we cannot distinguish claims about truth, semantics, or mental states solely by looking at internal properties of the individual; instead, we must consider the current and/or historical environment in which those claims are made and in which the current activity is taking place - suggest that the mind and cognitive processes "ain't (all) in the head!" Instead, they claim the mind can extend beyond the skull/skin boundary to loop into the body and environment.

The first wave is focused on functionalist accounts of mind that held the body and environment may be a constitutive part of cognition insofar as they play the right sorts of functional role for the cognitive system. If there is enough of a similar role for when a person uses their brain or when they turn to a trusty notebook to remember something, for instance, then both the brain and the notebook may be part of cognition. This similarity can be understood either in terms of folk psychological understandings of mental states or in terms of the most updated neuroscience, psychology, and other scientific theories. ${ }^{1}$ The second wave shifts to focus on how external objects and processes internal to the organism can be integrated without necessarily postulating an inherent isomorphism between internal and external (Menary, 2007; Sutton, Harris, Keil, \& Barnier, 2010). They instead focus on tracing ways that unique cognitive processes come about when an agent acts in the world. Finally, proponents of the third wave direct their attention to the specific dynamics and temporal components that were lacking in many examples in the first two waves. By this view, we may need to rethink the locus of agency in light of the complicated and continuous dance between brain, body, and world.

For an example of the first wave, we can consider the well-known Otto-Inga thought experiment as proposed by Clark and Chalmers (1998). In this case, both have the desire to attend an art exhibit at the Metropolitan Museum of Art (MoMA) in New York City. Based on this desire, Inga simply recalls the directions to the MoMA from memory and sets off to the correct address. Otto, unfortunately, suffers from early onset Alzheimer's, and therefore has difficulty with his biological memory. To compensate for such lapses, he carries around a notebook with relevant sorts of information, including, in this case, the location of the MoMA. After flipping through the notebook and finding the address, he successfully reaches the art exhibit. According to Clark and Chalmers, the information stored in Otto's notebook should be understood as dispositional beliefs in exactly the same functional way as the information stored in Inga's biological memory.

\footnotetext{
1 In a nutshell, functionalists argue that different parts of a cognitive architecture are best understood and individuated in light of the function each mental state plays for the cognitive economy of the system (e.g., a belief about something vs. a desire about something), without reducing them to their physical implementations or behavioral outputs. Folk psychological means that we make attributions of things like beliefs or desires based on how people use the terms in their everyday life, without necessarily deferring to what science tells us is "really" happening in the brain-body.
} 


\begin{tabular}{|c|c|c|c|}
\hline Wave & Principle & Example & References \\
\hline First & Parity & Otto-Inga thought experiment & $\begin{array}{c}\text { Clark \& Chalmers, 1998; } \\
\text { Wheeler, 2010 }\end{array}$ \\
\hline Second & Complementarity & Use of gestures in speech & $\begin{array}{c}\text { Menary, 2007; Sutton, } \\
2010\end{array}$ \\
\hline Third & Dynamic & Eve's Diary thought experiment & $\begin{array}{c}\text { Kirchoff, 2012; Colombetti } \\
\text { \& Roberts, 2015 }\end{array}$ \\
\hline
\end{tabular}

Fig. 1. Three waves of the Extended Mind Thesis.

Their conclusion rests on the parity principle, which states that "If, as we confront some task, a part of the world functions as a process which, were it done in the head, we would have no hesitation in recognizing as part of the cognitive process, then that part of the world is (so we claim) part of the cognitive process." (Clark \& Chalmers, 1998, p. 8, emphasis in original). In this case, since there is parity of function, even with differences for several features of the respective processes - e.g., Otto has to look at his notebook and the structure of the notebook information may not decay in the same sorts of ways as Inga's biological memory there is good reason to consider both the information stored in Inga's brain and the information stored in Otto's notebook as beliefs, and thus as proper parts of cognition.

The second wave developed an important shift in focus from parity to complementarity. When introducing this second wave, Sutton, for example, argues that "in extended cognitive systems, external states and processes need not mimic or replicate the formats, dynamics, or functions of inner states and processes. Rather, different components of the overall (enduring or temporary) system can play quite different roles and have different properties while coupling in collective and complementary contributions to flexible thinking and acting" (2010, p. 194). For instance, considering Otto, a second wave account rejects the use of functional similarity to ground cognitive extension. Instead, we ought to focus on how Otto's tight coupling to the notebook makes possible his memory and current action, instead of attempting to leverage an argument for functional similarity between Otto and Inga.

An additional example of complementarity involves gesture. While gestures are part of the body, they are nevertheless outside of the brain, and therefore extended beyond the skull boundary. Research conducted by Goldin-Meadow (e.g., Golden-Meadow, 2003; see also Clark, 2008, chapter 6 ) has found body movements that carried prima facie little or no physically practical effects can help or hinder various cognitive tasks. Furthermore, these effects come from the actual use of the hands, arms, fingers, and the like. In this case, the argument for the extended mind is not based on showing that external elements can function in similar ways to internal elements. A second wave theorist, after all, is not saying that gestures are part of cognition in these instances because they functionally mirror some neural processes. Rather, they attempt to establish how these external elements can complement (and facilitate) processes occurring internally to the agent's head.

In original motivations for the second wave, an additional concern was that parity may blind us to ways in which unique cognitive processes arise when we accept that cognition is not necessarily a brain-bound phenomenon. However, the second wave also displays its own shortcomings, which has prompted the development of a third wave. According to Kirchoff (2012), the focus on functional similarity of the first wave does not allow us to properly consider how extension transforms one's representational capacities. In other words, it does not explore how an organism's internal processing changes over time. For instance, learning how to read and use a tool, such as Otto using his notebook, is not just about linking up a bare, static brain to a bare, static tool. Likewise, using gestures is not just about linking brain and gestures together without any further changes to either. Both sides ulti- mately change during the process. In a similar manner, Sutton (2010) suggests that a third wave approach may push against the tendency for the second wave to hold the relevant internal and external properties as fixed throughout the development and execution of cognitive processes.

For an example of the third wave, following Colombetti and Roberts (2015), we can consider the case of extended occurrent emotional episodes. One possible feature of an emotional episode involves judgment, such as in the case of one judging that she or he is wronged as a constitutive feature of anger or judging that one has been hurt as a constitutive feature of sadness. Colombetti and Roberts argue that this judgment process may be extended by the use of an external device or tool, such as a diary. They offer the case of Eve, who regularly keeps a detailed diary that includes her feelings about her parents. After first arguing that the dispositional features of Eve's emotions are extended by the diary in a similar fashion to Otto's notebook, they turn to argue that occurrent instances of her emotion-constituting judgments are extended. In their words, "writing about one's emotion episodes is rarely (if ever) a mere description of one's evaluative position. More often, the act of writing, as it takes place, contributes to clarifying and articulating one's thoughts, to demarcating them more clearly from others, and thus to constraining the judging process as it is performed by the emoting subject" (2015, pp. 1257-1258, emphasis in original). To fully understand the nature of the emotional state, on this account, we must trace the diachronic development of the process. In doing so, the dynamics cannot be addressed in terms of parity. The judgment process is not extended simply because it mirrors a similar sort of internal process. Likewise, in contrast to a purely complementarity-based approach, it also focusses on how various engagements with the external world continuously reshape the contours of relevant mental processes and their properties. ${ }^{2}$

It is furthermore important to note the way in which the diary is a malleable yet permanent part of Eve's environment. Text can be added, altered, and deleted, but there is always a basis of existing text from which changes are made. A similar point has been raised in the literature on niche construction ${ }^{3}$ (Leland, Matthews, \& Feldman, 2016) and cultural affordances (e.g., Ramstead, Veissière, \& Kirmayer, 2016), as we will see in section 3 . In all these cases, humans find themselves in an environment that has already been shaped. Writing needed to exist before diaries could be in the form of books, for instance. At the same time, it's an environment that keeps changing according to how we actively engage with it. The development of emojis and text-message abbreviations are part of a changing environment of writing and commu-

\footnotetext{
2 As we will see in more details in section 2.2, the third wave extended mind accoun is particularly suited to capture the complex phenomenology of musical emotion, without reducing them to properties of either music or of listeners.

3 'Niche construction' is a term adopted by several schools of thought in evolutionary biology that are interested in how organisms can shape their environment across multiple levels and time scales. Moreover, these alterations will have looping effects that in turn change evolutionary selection pressures over time. For instance, a beaver building a dam alters the sorts of evolutionary pressures for itself, other organisms in the environment, and future organisms who will be around later as the dam remains in place.
} 
nication that also shapes the ways in which we can engage moving forward.

Learning and developing in such dynamic environments further modulates the sorts of processes utilized in day-to-day contexts, yet because the environment is never given a priori, it does not constrain unidirectionally the sorts of actions that can take place (see Thompson, 2007). For instance, it is common for a de facto trail to be created around the perpendicular intersection of two sidewalks if enough people start cutting the corner. Moreover, the more that people follow the new path, the clearer it becomes, and the more likely people will take the shortcut instead of staying on the sidewalks in the future. Said differently, then, acting on and within the environment, either to support what it already affords (I can walk along the sidewalks the whole time) or to change it (take the shortcut), strongly shapes the sorts of evolutionary and developmental pressure at play. It is through such bidirectional dynamicity that we can see how the third wave pushes against fixed properties and the assumption that the internal bits of the system will remain the same throughout time and location.

An enactivist reader may here wonder whether these claims simply collapse third wave extended mind into the enactivist framework. On one hand, we agree that there are important overlaps between the third wave extended mind and the extensive mind (Hutto, Kirchhoff, \& Myin, 2014), and there has been some critical work concerning whether it is possible to relate enactivist proposals with more conservative embodiment claims (Gallagher, 2017a; 2018). On the other hand, one major difference between the extended mind and extensive mind concerns how the larger cognitive system at play is not organism-centered for the extended mind in the ways that some enactivist frameworks imply.

As a result, we need to consider how agency is maintained or changed in these contexts. The focus on parity in the first wave or cognitive assembly and integration in the second wave is not enough to decentralize the notion of agency. It is important to decentralize this concept in the first place in order to address certain problematic assumptions from a centralized version. Agency is often understood as something that an individual has, either as a property or in regards to her capacity to intentionally act in the world. However, if cognition is extended, there is an important sense in which agency can be distributed across the larger cognitive systems that includes, but is not reducible to, individuals.

There are several different ways that this extension in agency can be understood. One way of understanding it is through the idea that there are active solicitations at play from various features of the environment (Rietveld \& Kiverstein, 2014). For instance, while my thirst may drive my desire to drink, there is something equally important about how a bottle of water solicits my response to take it and drink. Such distribution, in turn, is further augmented in the case of group activities, where the solicitations can come from others. Another possibility is to put pressure on the idea that agency is located purely within an individual. This pressure may come from the idea that agency can be extended to include things (Malafouris, 2013). Another alternative, following a long line of research in feminist philosophy, is the idea of relational autonomy that rejects an understanding of autonomy as fully individual (Westlund, 2009; Stoljar, 2019). Instead, autonomy can be only understood as having a fundamentally inter (en)active foundation (see also Gallagher, 2017b for a connection of feminism and enactivism on relational agency and interaction).

In summary, proponents of the first wave accept the parity principle (PP), while defenders of the second wave reject it and invoke instead a complementarity principle (CP). By this latter view, internal and external processes need not be functionally equivalent to be coupled, as supporters of PP argue. Instead, they only need to be complimentary to each other and operate together as an extended cognitive system (Menary, 2007; Sutton, 2010). Proponents of the third wave likewise maintain that elements external to one's physical brain and body can co-constitute the individual's mind by dynamically participating in the realization of a specific cognitive process. The third wave thus invokes what we consider to be a dynamic principle (DP). This DP is partially an extension of $\mathrm{CP}$ and pushes us to further examine specific features of dynamic processes as well as rethink the role and locus of agency during such processes.

With the basic distinctions among the three waves at play, we now turn to introduce their use in the context of musicking, especially in regards to musical emotions and music performance. While our ultimate aim is to explore how a third wave account of the extended mind may be beneficial to better capture the main features of human musicality and music-making experience, we begin by exploring how the first and second waves of the extended musical mind have already been developed.

\subsection{From musical emotion to music-specific extensions}

The current debate over the nature and properties of musical emotions is varied and highly significant in its broad range of approaches, methods, and results (see Cespedes-Guevara \& Eerola, 2018; Reybrouck \& Eerola, 2017; Reybrouck, Eerola, \& Podlipniak, 2018b). Though its complexity cannot be reduced to a particular issue, one important component of the discussion involves the distinction put forward by Gabrielsson (2002) between the categories of emotions expressed by the music, and emotions felt by the listener (see also Schubert, 2013). While the former focuses on the study of how emotion can be recognized within a given musical piece, scholars interested in the latter investigate how music induces an emotional response in the listener (see Cochrane, Fantini, \& Scherer, 2013). This distinction has informed the current debate in various ways, framing a number of important theoretical and empirical contributions (e.g., Fabian, Timmers, \& Schubert, 2014). From an extended mind perspective, however, distinguishing between these two categories might be problematic. In particular, as the third wave extended musical mind account emphasizes the dynamically interacting trajectories that musical emotions entail, it can eschew this dichotomy and offer a different understanding of musical emotions (for a similar move within a more enactive framework see Schiavio, van der Schyff, Cespedes-Guevara, \& Reybrouck, 2017a, b). In what follows we consider examples of first and second wave extended musical mind, and then offer preliminary insights for the development of a third wave account.

A good example of the first wave extended musical mind can be found in Tom Cochrane's work on emotion (2008). Cochrane specifically considers the role of emotions in music performance, which requires considerations about the intense connection between performer and emotion. He argues that there are certain cases where music itself may partially constitute a musician's emotional state during performance. This argument draws from functionalist considerations about the nature of emotions as well as a resemblance theory of music expression. According to defenders of the resemblance theory, "[v]ia a variety of resources (such as rhythm, timbre, melodic line), music is able to resemble the dynamic and visceral qualities of bodily feeling" (Cochrane, 2008, p. 329). In a nutshell, Cochrane first maintains that mental states are individuated and defined in terms of the role they play for a system, instead of their physical make-up. He then invites us to consider how an emotion is a combination of factors that includes a bodily pattern. These bodily patterns involve postures, gestures, and other behavioral expressions, which are also associated with particular states in the musician's brain. In certain cases (e.g., during performance), Cochrane notes that music can perform the same function as the bodily patterns that constitutes emotions in other contexts, primarily because it is able to resemble bodily patterns. Since music is external to the body then, accordingly, when these certain conditions are 
met, emotions may extend to include the music itself (see Kersten, 2017, pp. 1081-1082).

The role of parity is particularly clear here when discussing the main features of music and emotion. Since music can operate in the same functional way as bodily states to realize an emotion, especially in the neural representation of those respective states, Cochrane argues that we should accept music as playing a constitutive role for our emotions. The PP likewise holds that if we consider a process as if it were done in the head - here, the mapping of bodily patterns in the brain as features of emotion and the use of music in the same sort of emotional role during performance - the match between internal processes and external processes entails that both brain patterns and the music itself are part of cognition.

There are important ways in which Cochrane's position can be useful for moving away from traditional cognitivist assumptions about the relationship between emotion and music, yet there are equally important limitations to his focus on the PP as the grounds for an extended musical mind. First, even in cases where music may seem to take the lead, such as when a performer becomes absorbed and consciously lost in the music, Cochrane's account puts the focus primarily on internal processes instead of the connection between music and performer in that moment. Since the argument is based on functional parity between music and bodily patterns, what matters is what is located entirely in the individual's bodily pattern that set up the criteria in the first place. It therefore cannot account for how the dynamic unfolding of that emotion over time results in particular changes to the agent. Second, this perspective cannot help us account for how music performance may give rise to theretofore unexperienced emotions.

There is also a third concern with Cochrane's approach about agency. Insofar as parity rests on functional similarity, appealing solely to parity would have made it difficult to talk about agency spreading outside of the individual agent. Relying on parity therefore cannot account for any feelings of agentive decentralization, such as cases of deep absorption in individual or group performance or the decreasing of self-agency in certain cases of collective performance (see Pacherie, 2013). There are also related concerns that the sort of functionalism it entails is too course-grained - that is, unable to distinguish between specific emotions and their realizing mechanisms - to ground an extension of emotion and affect (cf. Kersten, 2017, p. 1089).

Another example of a first wave approach is Luke Kersten's (2014; 2017) Extended Computational Approach to Music Cognition (ECMC). ${ }^{4}$ While not explicitly functionalist in orientation, it shares important structural similarities to Cochrane's perspective and functionalism in general. According to the ECMC, we should first understand cognitive processes as computational processes. Computation, at least of a particular kind, thus acts as our benchmark for cognition. As a result, any sorts of processes that correctly realize these respective computations will be cognitive processes, even if they span outside the skull/skin boundary. While traditionally thought of as occurring in the head, there is nothing that logically requires any a priori limitation on the location of the computational processes that constitute cognition. As a result, since the physical realization of computations is open in terms of where they can occur, and assuming that there are instances of wide realization bases ${ }^{5}$ for musical cognition (Kersten \& Wilson, 2013), there are cases where musical cognition will be extended.

4 While both Cochrane and Kersten appeal to empirical work in defense of their claims, whether or not they take computation to be a mark of the mental helps distinguish their accounts. Cochrane's account can be developed with or without that assumption, while Kersten's account is computational through and through.

5 Realization base refers to the physical stuff over which a computation is performed. For a wide realization base, the relevant mental computations take place across the brain, body, and world. For a narrow realization base, in contrast, the computations take place purely inside the organism (e.g., neural computations).
A problem with the ECMC, at least as developed so far, is that it fails to account for the central role of affect and emotions in musical performance (cf. Krueger, 2014a). Kersten (2017) attempts to address this concern by appealing to the personal/subpersonal distinction (Dennett, 1969). He suggests that the ECMC is solely pitched at the subpersonal level and, insofar as this level is below conscious awareness, he claims that there is no need to appeal to emotions. While we grant that the personal/subpersonal distinction is an important one, we nevertheless suggest that Kersten's appeal to it in defense of the ECMC is not enough to address the role of emotions in music cognition.

There are at least two concerns with Kersten's current defense. First, he appears to conflate at least two different understandings of the personal/subpersonal distinction. This particular distinction is often invoked in making sense of how different psychological explanations are related. On one hand, it could rest on distinguishing processes that are accessible to consciousness (personal level) or not (subpersonal level). On the other hand, it could trace whether we are making attributions at the person level that includes a reliance on folk psychological concepts such as belief and desire attributed to the person as a whole (personal level; e.g., I have beliefs and desires; a section of my brain does not), or attributions about the underlying processes that may instead operate in terms amenable to mechanistic descriptions (subpersonal level; e.g., These neural components come together in cases of strong pleasure responses). Clarifying which of these understandings is at play will be important for both better understanding the ECMC as well as how it relates to alternative extended musical mind proposals. ${ }^{6}$

Second, we suggest that limiting the ECMC to a purportedly emotionless subpersonal level is problematic regardless of which aforementioned reading of the personal/subpersonal distinction one prefers. On a consciousness-based reading, we find that the emotions and affect operate in important ways at both levels, since there are times when we may be driven by emotions without being aware of them. Likewise, how emotions come to play their particular roles for music performance and perception cannot be limited to how they appear in consciousness, since important parts of emotion are realized in processes that operate unconsciously.

Alternatively, if we accept a folk psychology vs. mechanistic reading of the distinction, while we grant that it is the person who is experiencing an emotion, we also suggest that emotions are neither exhausted as commonsense constructs in folk psychology nor as propositional attitudes $^{7}$ and thus are not best understood as existing solely on the personal level. As a result, the mechanistic sorts of explanations at the subpersonal level are required to help flesh out the story of what is experienced by the person, especially in relation to emotions.

In contrast to first wave accounts, a second wave account that takes seriously the role of emotion and affect can be found in the work of Joel Krueger (2011; 2014a; 2014b). Krueger has argued that pairing with music and the use of musical affordances allows us to realize and manipulate emotional states in theretofore nonexistent ways. For instance, consider the case of being in a bad mood and wanting to feel better. To do so, you go to your playlist and put on your favorite song. Playing the music will allow you to manipulate and change your emotions. Moreover, while imagining your favorite song may be helpful, there is something distinctly important about actually listening to it.

\footnotetext{
6 Zoe Drayson (2012) provides an important clarification among different readings of the personal/subpersonal distinction, including and going beyond the two mentioned here She argues that it primarily traces a distinction among different types of psychological theory and, as a result, should be read in the second sense of folk-psychological explanations at the personal level and mechanistic explanations at the subpersonal level.

7 Propositional attitudes are a kind of mental state that relates the subject to a proposition. For instance, John believes that rain is wet is a combination of subject (John), mental state (believes), and a proposition (rain is wet)
} 
The music opens up a depth of emotion, and sometimes additional emotions, that comes about between the complementarity of the agent and the music in the world.

As a result, the integration between listener or performer and music requires us to re-center our empirical and theoretical focus on the larger agent-music whole. It is not enough to just study your musical taste or the structure of your favorite song to find out why listening to it makes you feel better. We need to focus on the music and the listener as one single system, instead of two distinct systems linking up. Likewise, the expanded contours of the emotional experiences that can be engaged with music are an essential part of Krueger's argument for the extended mind. As mentioned in the previous paragraph, there is something necessary about actually perceiving music that cannot be understood in isolation from engaging with it.

We can see complementarity at play in Krueger's work with this final point of how music makes possible certain emotions that would be otherwise unavailable to the agent. He suggests that this possibility exists because music offers novel affordances, which allow both a more active manipulation of emotions through the use of music as well as a richness to the phenomenological experience that cannot take place without actually listening. These affordances are part of a scaffolding process, since, in Krueger's words, "when we engage with music, we potentially access an expanded horizon of regulative possibilities that bring with them a richer palate of emotional expression and experience than we could access purely by appealing to our own internal (i.e., non-musically enhanced) resources" (2014b, p. 8). This connection often comes through forms of entrainment - e.g., a process where a person locks onto a beat, such as tapping along to a song - and is solicited by the contours of the musical sounds themselves. The emotional role of music is thus not just about subpersonal processing or standing in for bodily processes, but opening up new cognitive and emotional possibilities within one's phenomenological experience.

While there are certain elements of Krueger's framework that overlap with a third wave extended mind account, thus far there have been no explicit third wave accounts of musical cognition to our knowledge. Indeed, while the ultimate difference between Krueger's account and a third wave account may be more in degree than kind, there are several important differences along the way. The first one concerns the role of musical agents, which he often sees as explicitly 'using' music. While he accepts that there are times where the music may be a driving force, he often returns to the individual's ability to manipulate his or her inner (emotional) states through music. As a result, music is still defined in terms of being a technology for use and manipulation, which implies that the locus of agency remains within the individual. Second, Krueger's account is primarily aimed at music listening. This focus is not a problem with the theory, but it does limit how much it can be used to consider cases of musical performance, as we do below. As a result, even if our proposed third wave account may be more of an extension of Krueger's work than a refutation, it still goes beyond it in several ways. Moreover, third wave accounts can also help us characterize emotional engagements to music as dynamical processes that emerge within the fluid negotiation of meanings that each musical experience entails. As the sphere of emotionality cannot be easily detached from our everyday, valenced, coupling with the world (Colombetti, 2015; Maiese, 2011), and because this coupling involves the co-constitution of self and environment, emotional experiences associated with music are not reducible to their recognition in sound or their induction in the listener. Instead, they are found within the complex network of adaptive sense-making that defines living in general, and being a 'musical subject' in particular. As we will see in the next section, this is best understood when considering musical performance.

\section{Performance and third wave extended musical systems}

In this section we propose that adopting a third wave extended mind and its richer set of explanatory tools will help us fill in the gaps left by first and second wave accounts. As a concrete example, we consider specific aspects of how music is performed in light of Bill Bruford's (2018) Integrated Model of the Circulation of Meaning (IMCM). Our line of reasoning relies on the idea that musical performance is often shaped by cultural, affective, and physical features of the environment in ways that go beyond extended functionalism and cognitive assembly. As such, an approach that puts more emphasis on the dynamical and temporal interplay between biological and non-biological features of one's cognitive ecology is needed. In particular, we will explore how such situations are permeated by a moment-to-moment phenomenology that makes each performative experience unique. It will be argued that the contingent nature of our musical engagements might provide insights concerning the cognition of the musical event being enacted (Roholt, 2014; Salice, Høffding, \& Gallagher, 2017). It should nevertheless be noted that we will not be here developing an advanced account of musical improvisation, performance, or the creative aspects they entail. Instead, our aim is to offer a preliminary conceptual framework that may complement existing perspectives, and inspire future scholarship on these and related issues.

Exploring similar situations from a third wave extended mind perspective can generate important advantages both methodologically and in terms of content. In particular, it can be helpful for (i) capturing the role of a decentralized agency, and (ii) for explaining how the development of performance niches impact fundamental cognitive processing involved during the creation of music. Moreover, such an approach may offer a new context for extended mind theorists to test the internal coherence of their arguments in a systematic confrontation with the concrete practice of musicking. Let us begin with the role of agency.

\subsection{Decentralized agency}

Musicians perform in an ambience that has a particular meaning for them and for the audience; they usually employ physical tools for their musicking (e.g., musical instruments), often coupled with technological devices (e.g., mics); they are aware of the constraints of the performance setting (e.g., a jazz concert; a band rehearsal); and they are able to adapt themselves to the changing demands of such settings (e.g., when an audience in a rock concert is not particularly involved, or when the acoustics in a concert hall requires adjustments in the performative task). While a growing number of contributions have recently utilized work from 4E cognition to inspire richer understandings of the complex sets of neural, corporeal, and intersubjective factors in play during similar activities (e.g., De Jaegher \& Di Paolo, 2007; Gallagher, 2017a, 2017b; Schiavio, Biasutti, van der Schyff, \& Parncutt, 2018b; van der Schyff et al., 2018) it remains unclear how the musician's agency can be best described. Does it extend along with his or her cognitive ecology? Or does it remain intrinsically internal to the agent? Here the conceptual resources offered by a third wave account of the extended mind view may provide important benefits to adjudicate the issue.

There are several different ways in which we can understand agency as decentralized. The first, as explored above, concerns the ways in which agency and autonomy are fundamentally relational processes. The second is to consider a more active role to environmental stimulus and affordances. Finally, the third concerns decentralizing the phenomenology of agency. One way of understanding this last sense of decentralization can be drawn from the work of Elizabeth Pacherie (2013). According to Pacherie, a sense of individual agency is strongly predicated by the ability to accurately predict the outcomes of 
one's actions. These predictions are also mediated by various skills learned by the individual (Pacherie, 2013). One particularly salient skill is learning to play an instrument. Training to learn the skills associated with a new instrument entails a variety of neural and bodily changes (e.g., Gruhn \& Rauscher, 2008). There is evidence that these changes, especially large scale-neural changes, may also have an impact in other areas of cognition (see Hyde et al., 2009; Patel, 2008; Reybrouck, Vuust, \& Brattico, 2018a). These sorts of changes are ripe for explanation by the dynamic principle, at the same time as they put pressure on deriving a full-account from the likes of parity or complementarity alone.

The question of decentralized agency is further highlighted when we consider group performance. For example, it is possible that an unexpected particular accent of the hi-hat might emerge while performing a standard jazz pattern, capturing the attention of the other musicians. It may also capture the attention of the drummer, impacting the way in which they continue their musicking activities. Different drummers will react in a variety of ways: will they emphasize the accent even more as the performance unfolds, or will they try to compensate for it by adding novel accents to the bass drum, the snare, and so forth? Or will they simply ignore it and try to keep the pattern as uniform as previously thought? What strategy will they utilize to optimize the collective performance as other musicians participate? And what can be more appropriate for the performative setting where this takes place?

At the same time, the drummer's choices are not taking place in a vacuum. The saxophone player may take the accent to signify a change in tempo, launching the band forward into a different speed. The drummer will make different choices depending on who they are playing with on a given performance. A disapproving look from the other musicians in the stage will drive the choices made and the meaning of those choices for everyone involved. The possibilities of even this simple case are virtually infinite, and show how musical actions cannot be determined a priori, nor they are properly understood as fully individual products (Moran, 2017; Schiavio \& De Jaegher, 2017; Schiavio \& Høffding, 2015). Cultural, social, and contextual factors all contribute in defining and shaping one's musicking. But we must now ask in what sense is individual drumming not fully realized by the lone performer? The need for explaining these changes are equally pressing when we consider the development of performance practice across a longer period of time and may not be suitable for a purely complimentary-based approach.

One may nevertheless suggest that the first wave parity principle is equipped to account for decentralized agency. ${ }^{8}$ A parity-based argument may run as follows: first, if we assume that agency is internal to an agent, then there should be some sort of inner, functional, relationship that can be charted. Once we have this function, moreover, it is in principle possible to find parts of the world that play the same functional role and, therefore, agency would be extended.

The main reason that parity cannot serve this decentralization purpose is because it is unable to cope with how agency changes during performance. Such changes are not primarily nor ultimately grounded in some kind of functional similarity, but instead are focused on difference. While charting out all the detailed ways in which these changes occur is outside the scope of our current analysis, it is both a point of further research and a topic we plan to return to in the future.

\subsection{Performance niches}

There are a wide variety of different factors that impact a musical performance. Some arise in the physical spaces - fields, arenas, clubs,

\footnotetext{
8 Thank you to an anonymous reviewer for pushing us on this point.
}

concert halls, recording studios, and basements, to name just a few that have specific benefits and challenges for musicking. Others are related to expectations for the audience, as well as if there is a separate audience beyond the performing musicians. Still others are concerned with whether or not the music is the focus of performance (as in the case of concerts), serves as an accompaniment to something else (such as film music), or is a mix of both (such as certain religious rituals). ${ }^{9}$ It is also common to distinguish between expert and novice performance, which spans across the aforementioned factors.

Bill Bruford (2018), a highly-respected professional drummer who has played with the likes of groups such as Yes and King Crimson, as well as a theoretician, offers a series of insights to help clarify important aspects for music performance. Two of particular interest for our current project are the Integrated Model of the Circulation of Meaning (IMCM) $)^{10}$ and two modes of performance: 'functional' and 'compositional'. Focusing on the modes of performance first, 'functional' performance consists of performance that is heavily circumscribed by the will and desires of a party or parties other than the musician herself or himself, such as the case of a session performer who must only play directly from a prepared chart. In contrast, 'compositional' performance offers more freedom, such as the case of improvisation or when a musician is allowed to craft their own parts in a studio session. In Bruford's words, "Action at one pole [i.e. 'functional'], we might say, intends to supply the expected; action at the other [i.e. 'compositional'] intends to supply the unexpected" (2018, p. 38). While there are clear differences among these cases, it is also important to note that most instances of performance will be functional and compositional to varying degrees, rather than strictly either one or the other.

The distinction between 'functional' and 'compositional' is further woven into the fabric of the IMCM. This model is integrated insofar as it brings together the spheres of the larger musical culture, the individual performer's lived experience, and the mediated action that occurs between the two. The musical culture refers to the combined knowledge and ways in which performance is currently practiced. The lived experience refers to how each individual crafts and presents their unique playing style. Finally, the mediation process is concerned with how these two come together. Understanding how these three aspects are related will explain the nature and grounds of a given musical performance.

The overall musical culture both limits and offers certain possibilities for the performer, just as the environment opens up and forecloses certain possibilities for an organism in its environmental niche. On one hand, a performance is only possible because it takes place within a culture. On the other hand, especially when considering the case of expert musicians, each performance can also shape the overall musical culture. Indeed, a performance has the potential to make possible things that were in a certain sense impossible before. ${ }^{11}$ This sort of bidirectionality between performer and performance space is similar to the case of niche construction, especially if we consider a specific musical culture as the niche in question. Being completely outside of a musical culture isn't possible, just as it is impossible to sit entirely outside of all culture. Yet, at the same time, the existent culture doesn't overdeter-

\footnotetext{
9 These examples are certainly not meant to provide strict definitions of such events. Many other musical settings are possible where we simply cannot draw a precise distinction between roles, or individuate where the focus of performance is located.

10 Bruford considers the IMCM in the context of drum culture in particular. We believe that it can be extended to music at large and treat it accordingly (Thanks to an anonymous reviewer for pointing out this possibility).

11 Thanks to Eric Lewis (Personal Correspondence) for making this point in conversation, especially in regards to the development of jazz and jazz improvisation.
} 
mine all, or perhaps even many, of the choices made during performance.

This dynamical looping between the individual, material, and cultural environment is clearly in line with an embodied perspective writ-large (see Chemero, 2009; Fuchs, 2017). Bruford is explicit about this connection and makes a point of drawing on the work of John Dewey, an oft-cited influence for the embodied cognition community for his anti-dualistic views, among others in its defense. At the same time, he does not consider the extended cognition account in much detail. We are thus faced with the question of how exactly Bruford's conceptual tools relate with the various waves of the extended mind. In other words, how do the parity principle (PP), complimentary principle (CP), and the dynamic principle (DP) come into play in this particular case?

To understand the relation among music culture and individual performers, we suggest that an appeal to the first wave and the PP would serve little purpose, since the dynamic coupling between performer and music culture cannot be fully understood in terms of functional similarity. Should the same be said about second wave and its focus on the $\mathrm{CP}$ ? For one thing, in favor of invoking complementarity, the interaction between the individual performer and the larger musical culture is clearly a case where the cognition of the single musician is realized by processes that are not merely located within the individual agent. Describing the IMCM in relation to complementarity therefore seems like an important part of the explanation.

The second wave nevertheless does not offer much in the way of explaining how the environment and the individual change in light of the contextual factors that take place during a performance. What kind of temporal dynamics occur between the act of performance, the selection of the appropriate motor schema to perform, and the subsequent behavioral modifications? Musicians have to adapt to their own musicking demands - they must engage in a musically meaningful way to the open horizon of possibilities emerging from their musical actions. Likewise, engaging with these horizons is not just about linking up an existent internal and external structures, but about changing them as well.

There is an additional and important limitation for invoking a complementarity approach. On one reading, complementarity implies that the individual performer is linking up with something, in this case the music as performed, imagined, and predicted, and thus the cognitive process is being realized by a combination of prima facie internal (e.g., emotions) and external (e.g., musical actions) components. As was noted in Small's concept of musicking, however, we suggest that music is not a reified external object (see Reybrouck, 2001; 2015). It is instead an emergent process that is negotiated among brains, bodies, and world, and realized in real time with contextual performance dynamics, where instruments and individuals are understood as continuous with each other, and mutually incorporated (Schiavio \& van der Schyff, 2018). This process entails an understanding of performance that is not limited to the distribution between internal (e.g., homeostatic) and external (e.g., behavioral) patterns of individual performers, but instead across a much wider range of components, including instruments, other performers, and - in some cases - audience members as well.

In addition to these considerations, there is an important role for improvised activity across the entire 'functional-compositional' spectrum of expert performance that is relevant to consider for a third wave approach. Performers, regardless of which performance pole they are closer to at a given time, will draw on improvised sorts of activities, although constrained in different sorts of ways. Yet even with such constraints, improvisation entails a necessary component of time constrained exploration. Such exploration, moreover, is realized within a specific musical performance, wherein many parts of the performance cannot be controlled by any one performer. As such, a complete picture of the cognitive processes underlying an expert musician's performance will be responsive to the real time decisions of their fellow musicians. Even in the case of an improvised solo, various features of the environ- ment, such as audience feedback or lack thereof, will shape the decision-making process of the performer. In addition, how the performer experiences their agency in that situation will change in a variety of ways as well.

\section{Conclusion}

In this paper we have advanced the idea that a third wave extended mind approach can provide conceptual resources to capture the complexity of musical activities. In particular, when compared to other approaches inspired by the first and second waves, the third wave may be best suited to provide a richer understanding of what musical performance entails. After critically engaging with recent literature in the area and considering the groundbreaking work by Cochrane, Kersten, and Krueger, we turned to focus on some of the concrete dynamics of musicking, and showed how (at least) two of its main properties cannot be fully captured by these existing accounts. These properties involve the role of agency and the dynamic constitution of a performative niche. We also briefly considered several ways in which the third wave, in contrast, may help explain such musical dynamics.

In order to trace out the core differences among the three waves, we suggested that each can be associated with a different principle. The parity principle of the first wave focused on functional similarity to establish that there are cases where the mind can include processes outside the skull/skin boundary. The complementarity principle of the second wave captured situations where the flexible and dynamic coupling of different processes, both internal and external to the organism, accounts for cognitive capacities and processes. In contrast to parity, complementarity does not presuppose that extended cognitive processes must look functionally identical to internal cognitive processes. Finally, the dynamic principle of the third wave focused on the shifts that occur to underlying internal processes during development and cases of extended cognition, as well as shifts to agency that may be part of the extended mind.

At the same time as these three principles relate to each other, the extended mind cannot be taken in isolation from developments in other parts of 4E music cognition. From the embodied perspective, the body-in-action - in its dynamical interplay with the environment - becomes the main explanatory unit of musical experience (Iyer, 2002; Maes, Leman, Palmer, \& Wanderly, 2014; Schiavio \& Altenmüller, 2015). This move allows scholars to decenter their focus on the brain as the main source of music cognition to explore a much more distributed network of meaningful interactivities involving the entire body of the agent (see Lakoff \& Johnson, 1980). When considering embedded cognition, the cultural and material ecology surrounding musical beings is emphasized (Clarke, 2005), giving rise for example to diverse understandings of musical affordances (Krueger, 2011; Menin \& Schiavio, 2012). Scholars working within an enactive approach defend the view that musical bodies, musical minds, and musical environments are co-constructed by their circular and self-sustaining dynamics (Laroche \& Kaddouch, 2015; Schiavio, van der Schyff, Kruse-Weber, \& Timmers, 2017b; van der Schyff \& Schiavio, 2017a). Finally, conceiving of the mind as an extended phenomenon, as we have explored in detail, implies that cognitive processes may use cultural and physical resources internal and external to the agent, such that aspects of mental life can be offloaded onto the environment in which one is situated, facilitating a number of musical-related tasks (Cochrane, 2008; Krueger, 2013, 2014a; Kersten, 2014; 2017). The extended mind in general has also been usefully adopted in other domains - for example in research involving emotions (Colombetti \& Roberts, 2015), artificial intelligence (Clark, 2001), evolutionary robotics, animal cognition (Japyassú \& Laland, 2017), and music education (Schiavio et al., 2018a, b). 
Further work on the extended mind, in continuity with the other Es, may also contribute additional benefits to our overall understanding of music cognition, and musical emotion - further elaborating the preliminary considerations offered in section 2.2. Concerning practicing musicians, an extended mind orientation might contribute to better understand their continuous negotiation between internal and external factors, as well as how emotional, social, and cultural dynamics, shape performance at different levels and timescales.

\section{Uncited references}

Froese et al., 2013, Juslin and Laukka, 2004, Wheeler, 2010

\section{Acknowledgments}

We would like to thank Shaun Gallagher and Richard Parncutt for comments and suggestions that improved our original draft. We would also like to thank the audience at the University of Memphis PGSA Brown Bag Series, as well as the editor and two anonymous reviewers for additional feedback. A.S. is supported by a Lise Meitner Postdoctoral Fellowship granted by the Austrian Science Fund (FWF): project number M2148.

\section{References}

Blacking, J., 1973. How musical is man?. Faber, London.

Borgo, D., 2005. Sync or swarm: Improvising music in a complex age. Continuum.

Bruford, B., 2018. Uncharted: Creativity and the expert drummer. U. Michigan Press, Ann Arbor.

Cespedes-Guevara, J., Eerola, T., 2018. Music communicates affects, not basic emotions a constructionist account of attribution of emotional meanings to music. Frontiers in Psychology 9, 215. https://doi.org/10.3389/fpsyg.2018.00215.

Chemero, A., 2009. Radical embodied cognitive science. MIT Press, Cambridge, MA.

Clark, A., 2001. Mindware: An introduction to the philosophy of cognitive science. Oxford U. Press, New York.

Clark, A., 2008. Supersizing the mind: Embodiment, action, and cognitive extension. Oxford U. Press, New York.

Clark, A., Chalmers, D., 1998. The extended mind. Analysis 58, 7-19.

Clarke, E., 2005. Ways of listening: An ecological approach to the perception of musical meaning. Oxford U. Press, New York.

Clarke, E., Doffman, M. (Eds.), 2017. Distributed creativity: Collaboration and improvisation in contemporary music. Oxford University Press, New York.

Clayton, M., 2017. The ethnography of embodied musical interaction. In: Lesaffre, M., Leman, M., Maes, P.J. (Eds.), The routledge companion to embodied music interaction. Routledge, New York, NY, pp. 215-222.

Cochrane, T., 2008. Expression and extended cognition. The Journal of Aesthetics and Art Criticism 66, 59-73.

Cochrane, T., Fantini, B., Scherer, K.R., 2013. The emotional power of music. Oxford University Press, Oxford.

Colombetti, G., Roberts, T., 2015. Extending the extended mind: The case for extended affectivity. Philosophical Studies 172, 1243-1263.

De Jaegher, H., Di Paolo, E.A., 2007. Participatory sense-making: An enactive approach to social cognition. Phenomenology and the Cognitive Sciences 6, 485-507. https://doi. org/10.1007/s11097-007-9076-9.

De Souza, J., 2017. Music at hand: Instruments, bodies, and cognition. Oxford U. Press, New York.

Dennett, D.C., 1969. Content and consciousness. Humanities Press, Oxford, UK.

Drayson, Z.-, 2012. The uses and abuses of the personal/subpersonal distinction. Philosophical Perspectives 26, 1-18.

Elliott, D., Silverman, M., 2015. Music matters: A philosophy of music education, $2^{\text {nd }}$. Oxford U. Press, New York.

Fabian, D., Timmers, R., Schubert, E., 2014. Expressiveness in music performance: Empirical approaches across styles and cultures. Oxford University Press, Oxford.

Froese, T., Gershenson, C., Rosenblueth, D.A., 2013. The dynamically extended mind - a minimal modeling case study, arXiv:1305.1958 [nlin]. Retrieved from http://arxiv. org/abs/1305.1958.

Fuchs, T., 2017. Ecology of the brain: The phenomenology and biology of the embodied mind. Oxford University Press, Oxford, UK.

Gabrielsson, A., 2002. Perceived emotion and felt emotion: Same or different?. Musicae Scientiae 6, 123-148.

Gallagher, S., 2017a. Enactivist interventions: Rethinking the mind. Oxford U. Press, Oxford.

Gallagher, S., 2017b. Social interaction, autonomy and recognition. In: Dolezal, L., Petherbridge, D. (Eds.), Body/Self/Other: The phenomenology of social encounters. Routledge, London, pp. 133-160.
Gallagher, S., 2018. Building a stronger concept of embodiment. In: Newen, A., de Bruin, L., Gallagher, S. (Eds.), The oxford handbook of cognition: Embodied, enactive, extended. Oxford U. Press, Oxford.

Goldin-Meadow, S., 2003. Hearing gesture: How our hands help us think. Harvard U. Press, Cambridge, MA.

Gruhn, W., Rauscher, F.H., 2008. Neurosciences in music pedagogy. Nova Science Pub Inc, New York, NY.

Hayes, L., 2017. From site-specific to site-responsive: Sound art performances as participatory milieux. Organised Sound 22, 82-92.

Himberg, T., 2017. Entrainment and mutual adaptation in musical movement and dance. In: Lesaffre, M., Leman, M., Maes, P.J. (Eds.), The routledge companion to embodied music interaction. Routledge, New York, NY, pp. 141-149.

Hutto, D.D., Kirchhoff, M.D., Myin, E., 2014. Extensive enactivism: Why keep it all in?. Frontiers in Human Neuroscience 8, 706.

Hyde, K., Lerch, J., Norton, A., Forgeard, M., Winner, E., Evans, A.C., et al., 2009. Musical training shapes structural brain development. Journal of Neuroscience 26 (10), 3019-3025.

Iyer, V., 2002. Embodied mind, situated cognition, and expressive microtiming in african-American music, music perception. An Interdisciplinary Journal 19, 387-414.

Japyassú, H.F., Laland, K.N., 2017. Extended spider cognition. Animal Cognition 20 (3), 375-395. https://doi.org/10.1007/s10071-017-1069-7.

Juslin, P.N., Laukka, P., 2004. Expression, perception, and induction of musical emotions: A review and a questionnaire study of everyday listening. Journal of New Music Research 33, 217-238. https://doi.org/10.1080/0929821042000317813.

Kersten, L., 2014. Music and cognitive extension. Empirical Musicology Review 9, 193-202.

Kersten, L., 2017. Extended Music Cognition Philosophical Psychology 30, 1078-1103.

Kersten, L., Wilson, R.A., 2013. The sound of music: Externalist style. American Philosophical Quarterly 53, 139-154.

Kirchhoff, M., 2012. Extended cognition and fixed properties: Steps to a third-wave version of extended cognition. Phenomenology and the Cognitive Sciences 11, 287-308.

Kozak, 2015. Listeners' bodies in music analysis: Gestures, motor intentionality, and models. Music Theory Online 21, 3.

Krueger, J., 2011. Doing things with music. Phenomenology and the Cognitive Sciences $10,1-22$.

Krueger, J., 2013. Empathy, enaction, and shared musical experience: Evidence from infant cognition. In: Cochrane, T., Fantini, B., Scherer, K. (Eds.), The emotional power of music: Multidisciplinary perspectives on musical expression, arousal and social control. Oxford U. Press, pp. 177-196.

Krueger, J., 2014a. Musical manipulations and the emotionally extended mind. Empirical Musicology Review 9, 208-212.

Krueger, J., 2014b. Affordances and the musically extended mind. Frontiers in Psychology 4 (1003), 1-12.

Krueger, J., 2015. Musicing, materiality, and the emotional niche. Action, Criticism and Theory For Music Education 14, 43-62.

Krueger, J., 2018. Music as affective scaffolding. In: Clarke, D., Herbert, R., Clarke, E. (Eds.), Music and consciousness II: Philosophical, psychological, and cultural perspectives. Oxford U. Press, Oxford.

Lakoff, G., Johnson, M., 1980. Metaphors we live by. University of Chicago Press, Chicago, $\mathrm{IL}$.

Laroche, J., Kaddouch, I., 2015. Spontaneous preferences and core tastes: Embodied musical personality and dynamics of interaction in a pedagogical method of improvisation. Frontiers in Psychology 6, 522

Leland, K., Matthews, B., Feldman, M.W., 2016. An introduction to niche construction theory. Evolutionary Ecology 30, 191-202.

Leman, M., 2007. Embodied music cognition and mediation technology. MIT Press, Cambridge, MA.

Loaiza, J., 2016. Musicking, embodiment and participatory enaction of music: Outline and key points. Connection Science 28, 1-13.

Maes, P.-J., Leman, M., Palmer, C., Wanderly, M., 2014. Action-based effects on music perception. Frontiers in Psychology 4, 1008. https://doi.org/10.3389/fpsyg.2013.01008.

Malafouris, L., 2013. How things shape the mind: A theory of material engagement. MIT Press, Cambridge, MA.

Menary, R., 2007. Cognitive integration: Mind and cognition unbounded. Palgrave Macmillian.

Menary, R., 2010. The extended mind. MIT Press, Cambridge, MA.

Menin, D., Schiavio, A., 2012. Rethinking musical affordances. AVANT. Trends in interdisciplinary studies 3 (2), 202-215.

Moran, N., 2014. Social implications arise in embodied music cognition research which can counter musicological "individualism.". Frontiers in Psychology 5, 676.

Moran, N., 2017. Agency in embodied music interaction. In: The routledge companion to embodied music interaction. Routledge, New York, NY, pp. 31-39.

Pacherie, E., 2013. How does it feel to act together?. Phenomenology and the Cognitive Sciences 13, 25-46.

Parncutt, R., McPherson, G. (Eds.), 2002. The science \& psychology of music performance. Oxford University Press, Oxford.

Patel, A.D., 2008. Music, language, and the brain. Oxford University Press, New York, NY.

Ramstead, M.J.D., Veissière, S.P.L., Kirmayer, L.J., 2016. Cultural affordances: Scaffolding local worlds through shared intentionality and regimes of attention. Frontiers in Psychology

Reybrouck, M., 2001. Biological roots of musical epistemology: Functional Cycles, Umwelt, and enactive listening. Semiotica 134 (1-4), 599-633. 
Reybrouck, M., 2005. A biosemiotic and ecological approach to music cognition: Event perception between auditory listening and cognitive economy. Axiomates 15, 391-409. https://doi.org/10.1007/s10516-004-6679-4.

Reybrouck, M., 2012. Musical sense-making and the concept of affordance: An ecosemiotic and experiential approach. Biosemiotics 5, 391-409. https://doi.org/10.1007/ s12304-012-9144-6.

Reybrouck, M., 2015. Real-time listening and the act of mental pointing: Deictic and indexical claims. Mind, Music, and Language 2, 1-17.

Reybrouck, M., Eerola, T., 2017. Music and its inductive power: A psychobiological and evolutionary approach to musical emotions. Frontiers in Psychology 8, 494. https:// doi.org/10.3389/fpsyg.2017.00494.

Reybrouck, M., Eerola, T., Podlipniak, P., 2018b. Editorial: Music and the functions of the brain: Arousal, emotions, and pleasure. Frontiers in Psychology 9, 113. https://doi. org/10.3389/fpsyg.2018.00113.

Reybrouck, M., Vuust, P., Brattico, E., 2018a. Music and the plastic brain: How sounds trigger neurogenerative adaptations. In: Chaban, V. (Ed.), Neuroplasticity: Insights of neural reorganization. InTech, Rijeka, pp. 85-103.

Rietveld, E., Kiverstein, J., 2014. A rich landscape of affordances. Ecological Psychology 26, 325-352.

Roholt, T., 2014. Groove: A phenomenology of rhythmic nuance. Bloomsbury, New York, NY.

Salice, A., Høffding, S., Gallagher, S., 2017. Putting plural self-awareness into practice: The phenomenology of expert musicianship. Topoi https://doi.org/10.1007/s11245017-9451-2.

Schiavio, A., Altenmüller, E., 2015. Exploring music-based rehabilitation for Parkinsonism through embodied cognitive science. Frontiers in Neurology 6, 217.

Schiavio, A., Biasutti, M., van der Schyff, D., Parncutt, R., 2018b. A matter of presence. A qualitative study on teaching individual and collective music classes. Musicae Scientiae https://doi.org/10.1177/1029864918808833, online first.

Schiavio, A., De Jaegher, H., 2017. Participatory sense-making in joint musical practices. In: Lesaffre, M., Leman, M., Maes, P.J. (Eds.), The routledge companion to embodied music interaction. Routledge, New York, NY, pp. 31-39.

Schiavio, A., Høffding, S., 2015. Playing together without communicating? A pre-reflective and enactive account of joint musical performance. Musicae Scientiae 19, 366-388.

Schiavio, A., Menin, D., Matyja, J., 2015. Music in the flesh. Embodied simulation in musical understanding. Psychomusicology: Music, Mind, \& Brain 24 (4), 340-343.

Schiavio, A., van der Schyff, D., 2018. 4E music cognition and the principles of self-organization. Behavioral Sciences 8 (8), 72.

Schiavio, A., van der Schyff, D., Cespedes-Guevara, J., Reybrouck, M., 2017a. Enacting musical emotions: Sense-making, dynamic systems, and the embodied mind. Phenomenology and the Cognitive Sciences 16 (5), 785-809.

Schiavio, A., van der Schyff, D., Gande, A., Kruse-Weber, S., 2018a. Negotiating individuality and collectivity in community music: A qualitative case study. In: Psychology of music. Advance online publicationhttps://doi.org/10.1177/0305735618775806.
Schiavio, A., van der Schyff, D., Kruse-Weber, S., Timmers, R., 2017b. When the sound becomes the goal: 4E cognition and teleomusicality in early infancy. Frontiers in Psychology 8 (1585)https://doi.org/10.3389/fpsyg.2017.01585.

Schubert, E., 2013. Emotion felt by the listener and expressed by the music: Literature review and theoretical perspectives. Frontiers in Psychology 4, 837. https://doi.org/10. 3389/fpsyg.2013.00837.

van der Schyff, D., Schiavio, A., 2017a. Evolutionary musicology meets embodied cognition. Biocultural coevolution and the enactive origins of human musicality. Frontiers in Neuroscience 11, 519. https://doi.org/10.3389/fnins.2017.00519.

van der Schyff, D., Schiavio, A., 2017b. The future of musical emotions. Frontiers in Psychology 8, 988. https://doi.org/10.3389/fpsyg.2017.00988.

van der Schyff, D., Schiavio, A., Elliott, D., 2016. Critical ontology for an enactive music pedagogy. Action. Criticism \& Theory for Music Education 15 (5), 81-121. https:// doi.org/10.22176/act15.5.81.

van der Schyff, D., Schiavio, A., Walton, A., Velardo, V., Chemero, T., 2018. Musical creativity and the embodied mind. Exploring the possibilities of $4 \mathrm{E}$ cognition and dynamical systems theory. Music \& Science https://doi.org/10.1177/205920431879231, online first.

Small, C., 1998. Musicking: The meanings of performing and listening. Wesleyan U. Press.

Stoljar, N., 2019. Feminist perspectives on autonomy (in press). Zalta, E.N. (Ed.), The stanford encyclopedia of philosophy. (Winter 2018 ed.). https://plato.stanford.edu/ archives/win2018/entries/feminism-autonomy.

Sutton, J., 2010. Exograms and interdiscipinarity: History, the extended mind, and the civilizing process. In: Menary, R. (Ed.), The extended mind. MIT Press, Cambridge, MA, pp. 189-225.

Sutton, J., Harris, C.B., Keil, P.G., Barnier, A.J., 2010. The psychology of memory, extended cognition, and socially distributed remembering. Phenomenology and the Cognitive Sciences 9, 521-560.

Thompson, E., 2007. Mind in life: Biology, phenomenology, and the sciences of mind. Harvard U. Press.

Walton, A., Richardson, M.J., Langland-Hassan, P., Chemero, A., 2015. Improvisation and the self-organization of multiple musical bodies. Frontiers in Psychology 1, 313. https: //doi.org/10.3389/fpsyg.2015.00313.

Westlund, A.C., 2009. Rethinking relational autonomy. Hypatia 24, 26-49.

Whalley, J.H., Mavros, P., Furniss, P.(, 2014. Clasp together: Composing for mind and machine. Empirical Musicology Review 9, 263-276.

Wheeler, M., 2010. In defense of extended functionalism. In: Menary, R. (Ed.), The extended mind. MIT Press, Cambridge, MA, pp. 245-270.

Williamon, A., 2004. Musical excellence. Strategies and techniques to enhance performance. Oxford University Press, Oxford-New York. 